Military Technical College Kobry El-Kobba Cairo, Egypt

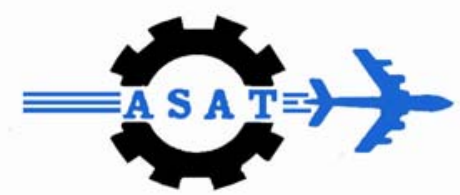

12-th International Conference on Aerospace Sciences \& Aviation Technology

\title{
ANALYSIS AND TRADE-OFF STUDY OF SIGNAL-TO-NOISE RATIO DEPENDENT DESIGN PARAMETERS OF SATELLITE PASSIVE ELECTRO-OPTICAL REMOTE SENSORS
}

Fawzy El-Tohamy Hasan*, Mahmoud Fathy Mahmoud**, Ibrahim Aly Abdel Dayem*, Adel Moharam El-Nozahy ${ }^{* * *}$ and Mohamed El-Sayed Abdel Hady*

\begin{abstract}
One of the main goals that is concerned to remote sensor designers is the quality of the produced image. There are a number of parameters to evaluate the quality of the image produced by an imaging sensor. The parameters widely used are the Modulation Transfer Function (MTF) and signal-to-noise ratio $(\mathrm{S} / \mathrm{N})$, for passive electro-optical remote sensors. In this paper an analysis and trade-off study of $\mathrm{S} / \mathrm{N}$ dependent sensor design parameters are presented.
\end{abstract}

KEY WORDS: remote sensing, signal-to-noise ratio, imaging modes, integration time, electro-optical remote sensors, design parameters.

* Egyptian Armed Forces

** Banha High Technology Institute, Banha, Egypt.

*** National Laser Institute, Cairo University, Giza, Egypt. 


\section{1- INTRODUCTION}

The $\mathrm{S} / \mathrm{N}$ is an important parameter, which decides the quality of the image. The $\mathrm{S} / \mathrm{N}$ depends directly on the noise characteristics of the detector used and the energy reaching the detector [1], which in turn depends on many sensor design parameters. The power, $\Phi[$ Watt], received at the detector is given by [2],

$$
\phi=\frac{\pi}{4} O_{e} \Delta \lambda L_{\lambda} \beta^{2} D^{2}
$$

Where, $L_{\lambda}$ is the scene spectral radiance [Watt $\left.m^{-2} S r^{-1} \mu m^{-1}\right], \Delta \lambda$ is the spectral bandwidth $[\mu \mathrm{m}], D$ is the diameter of the collecting optics $[\mathrm{m}], \beta$ is the instantaneous field of view [rad], and $O_{e}$ is the optical efficiency (optical transmission).

If the scene is observed for a time $\tau$ seconds (usually called dwell time or integration time), then the energy, $Q$ [Joule], collected by the detector is given by [3],

$$
Q=\frac{\pi}{4} O_{e} \Delta \lambda L_{\lambda} \beta^{2} D^{2} \tau
$$

From equation (2) it is clear that the energy colleted is proportional to the following sensor parameters: the optical aperture area, the Instantaneous field of view (which reflects the spatial resolution), signal integration time, and spectral bandwidth. However the change of one of these parameters to improve an aspect, for example, for improving high spatial resolution, (i.e., minimizing the ground resolved distance - GRD), $\beta$ has to be reduced, to keep the same energy $Q$ this will be at the expense of other aspects. For example, the optics diameter $D$ should be increased, wherein optical size and weight increase, or the spectral bandwidth $\Delta \lambda$ should be increased, which means that the spectral resolution has to be compromised, the integration time $\tau$ should be increased, which means choice of another sensor type (i.e., another imaging mode) [4]. Therefore $\mathrm{S} / \mathrm{N}$ provides a useful framework for examining the interdependencies among sensor performance and design parameters. This inter-relationship between the various sensor parameters sets a limit on sensor performance, in other words realizing the best possible performance (all the resolutions achievable from a sensor) critically influences the complexity of the sensor.

\section{2- INTEGRATION TIME AND IMAGING MODES}

From equation (2) we see that the energy colleted is proportional to integration time $\tau$ (among other parameters), therefore the sensor designer tries to maximize this time within practical engineering limitations, as a solution when reducing $\beta$ to achieve high spatial resolution. In fact, maximizing the integration time is an important consideration for the choice of the sensor imaging technique (or sensor type). There is a considerable difference in integration time among the available imaging techniques in remote sensing. From this point of view, the most common imaging techniques used today in remote 
sensing satellites can be classified into three modes, Fig.1: whiskbroom, (optomechanical scanners), normal pushbroom, and time-delay-and integration (TDI) technique [4].

i) Whiskbroom mode (Opto-mechanical scanner): In this mode, Fig.1a, the sensor collects the radiation from one ground pixel at a time. A scan mirror directs the sensor to the next pixel in the cross-track direction and by the scan mirror motion, one cross-track line of width equal to one pixel is imaged. Successive scan lines are produced by the motion of the platform. Dwell time is relatively small in this imaging mode. Typical sensors utilizing this mode of imaging include LANDSAT Multi-Spectral Scanner (MSS), Thematic Mapper (TM) and Enhanced Thematic Mapper (ETM) [5, 6].

ii) Normal pushbroom mode: In this mode, Fig. $1 \mathrm{~b}$, the sensor collects radiation from one line in the cross-track direction at one instant, without mechanical scanning. Successive lines are scanned by platform motion. Linear (1-D) detector array generates images using this mode. Some typical sensors utilizing this mode of imaging include SPOT-4 High Resolution Visible camera (HRV); and Indian Remote Sensing (IRS) linear imaging self-scanning sensors (LISS- I, II, and III), and IRS-1C PAN camera [5, 6].

iii) Time-delay-and integration (TDI): In this mode, Fig.1c, multi-linear arrays are used instead of a single linear array used in the normal pushbroom mode, for more increasing of the dwell time. IKONOS-1 satellite sensor is a typical example using the TDI imaging mode $[5,6]$.

By comparing these imaging modes, we find a considerable difference in integration time, which is important for the choice of a scanner type.

\section{2-1 Comparing an Opto-mechanical Scanner with a Normal Pushbroom}

Let $T_{o}$ be the integration time for an opto-mechanical scanner (having a single detector element per band) and $\tau_{p}$ the integration time for a normal pushbroom scanner, all other parameters remaining the same, then $\tau_{p} / T_{o}=\Omega / \beta S_{\text {eo }}$ [2], where $\Omega$ is the total field of view (TFOV) [rad], and $S_{e o}$ is the scan efficiency of an opto-mechanical scanner. $\Omega / \beta$ is the number of elements on the detector array (across-track pixels). Thus the integration time is considerably larger in a normal pushbroom scanner compared to an opto-mechanical one.

\section{2-2 Comparing a Normal Pushbroom Scanner with TDI}

Consider a normal pushbroom scanner a single detector array consisting of $P$ pixels (across-track) and a TDI scanner with $N$ rows (along-track), of the same detector array of $P$ pixels, then the charge accumulated in each row is transferred to the successive lines at a rate exactly compensating the image motion, thereby increasing the effective 
integration time $N$ times of normal pushbroom and also, increasing the $\mathrm{S} / \mathrm{N}$ by a factor of $\sqrt{N}[7,8]$.

\section{3- S/N AND SCANNERS DESIGN PARAMETERS}

For an opto-mechanical scanner with photodiode detectors, the $S / N$ is given by $[1,6]$,

$$
S / N \propto \frac{D^{2} \beta^{3} n^{0.5}}{\Omega^{0.5}}
$$

Where, $n$ is the number of along-track detector elements.

For a normal pushbroom scanner of one linear photodiode detector array, $S / N$ is improved, with respect to the opto-mechanical scanner, by the factor $\sqrt{P}[7,8]$. In this case $P=\Omega / \beta$, for pushbroom scanner the $\mathrm{S} / \mathrm{N}$ is such that:

$$
\begin{gathered}
S / N \propto \frac{D^{2} \beta^{3}}{\sqrt{\Omega}} \sqrt{\frac{\Omega}{\beta}} \\
\therefore S / N \propto D^{2} \beta^{2.5}
\end{gathered}
$$

For a TDI pushbroom scanner containing $N$ linear photodiode along-track detector arrays, the $\mathrm{S} / \mathrm{N}$ will be improved by the factor $\sqrt{N}$ with respect to normal pushbroom one containing a single linear detector array. In this case the $S / N$ is such that:

$$
S / N \propto D^{2} \beta^{5 / 2} \sqrt{N}
$$

One of the major parameters which describe the image quality is the ground resolution. We make a comparison to illustrate how specific ground resolution values can be realized by the three imaging techniques of scanners and the dependence of this ground resolution on the optical diameter as an important design parameter.

\section{4- PARAMETERS OF SELECTED SCANNERS}

Some examples of opto-mechanical, normal pushbroom, and TDI pushbroom scanners of similar detector types and scan characteristics are selected for analysis and trade-off study of $\mathrm{S} / \mathrm{N}$ dependent scanners design parameters.

An example of opto-mechanical scanner used today is the Enhanced Thematic Mapper plus $(E T M+)$ in LandSat-7 orbiting at altitude $705 \mathrm{Km}$, this scanner realizes $15 \mathrm{~m}$ ground 
resolution in pan-chromatic band $(0.52-0.9) \mu \mathrm{m}[3,6]$. The design parameters of ETM+ scanner are given in Table 1.

Table1. Design parameters of ETM+ opto-mechanical scanner

\begin{tabular}{llcc}
\hline $\mathbf{S}$ & \multicolumn{1}{c}{ Design parameter } & Symbol & ETM+ \\
\hline 1 & Entrance aperture diameter [cm] & $D$ & 40.64 \\
2 & Instantaneous field of view [urad] & $B$ & 21.277 \\
3 & Number of along-track detector pixels & $N$ & 32 \\
4 & Total field of view [rad] & $\Omega$ & 0.26 \\
\hline
\end{tabular}

Examples of normal Pushbroom scanners used today are HRV, in SPOT-4, and PAN camera, in IRS-1C satellites orbiting at altitudes 822 and $816 \mathrm{Km}$ respectively. The HRV realizes $10 \mathrm{~m}$ ground resolution in pan-chromatic band $(0.51-0.73 \mu \mathrm{m})$, while the PAN camera realizes $5.8 \mathrm{~m}$ ground resolution in the pan-chromatic band $(0.5-0.75 \mu \mathrm{m})[3$, 6]. The design parameters of these scanners are given in Table 2.

Table 2. Design parameters of SPOT-4 HRV and IRS-1C PAN pushbroom scanners

\begin{tabular}{|c|c|c|c|c|}
\hline $\mathbf{S}$ & Design parameter & Symbol & $\begin{array}{c}\text { SPOT-4 } \\
\text { HRV }\end{array}$ & $\begin{array}{c}\text { IRS-1C } \\
\text { PAN }\end{array}$ \\
\hline 1 & Entrance aperture diameter $[\mathrm{cm}]$ & $D$ & 30.91 & 22 \\
\hline 2 & Instantaneous field of view [ $\mu \mathrm{rad}]$ & $B$ & 12.165 & 7.1 \\
\hline 3 & Number of cross-track detector pixels & $P$ & $1 \times 6000$ & $3 \times 4096$ \\
\hline 4 & Total Field of view [rad] & $\Omega$ & 0.073 & 0.077 \\
\hline
\end{tabular}

An example of TDI Pushbroom scanner is that used today in the IKONOS-1 satellite orbiting at altitude $681 \mathrm{Km}$. It realizes $1 \mathrm{~m}$ ground resolution in the band $(0.45-0.9 \mu \mathrm{m})$ $[3,6]$. The design parameters of this scanner are given in Table 3. 
Table 3. Design parameters of TDI pushbroom scanner in IKONOS satellite

\begin{tabular}{clcc}
\hline $\mathbf{S}$ & Design parameter & Symbol & $\begin{array}{c}\text { IKONOS-1 } \\
\text { TDI }\end{array}$ \\
\hline 1 & Entrance aperture diameter [cm] & $D$ & 70 \\
2 & Instantaneous field of view [urad] & $\beta$ & 1.2 \\
3 & Number of along-track detector arrays & $N$ & 32 \\
4 & Total Field of view [rad] & $\Omega$ & 0.016 \\
\hline
\end{tabular}

\section{5- DEPENDENCE OF INSTANTANEOUS FIELD OF VIEW ON ENTRANCE APERTURE DIAMETER}

\subsection{In Case of Opto-mechanical and Normal Pushbroom Scanners}

From relation (3), we can write: $\quad D=\frac{(\text { Const } .) \Omega^{1 / 4}}{\beta^{3 / 2} n^{1 / 4}}$

So, in case of LandSat-7 ETM+ $\quad D=\frac{3.99 \times 10^{-8}}{\beta^{3 / 2}}$

From relation (4) we can write: $\quad D=\frac{(\text { Const })}{\beta^{5 / 4}}$

So, in case of SPOT-4 HRV

$$
D=\frac{2.22 \times 10^{-7}}{\beta^{5 / 4}}
$$

and in case of IRS-1C PAN camera $D=\frac{8.106 \times 10^{-8}}{\beta^{5 / 4}}$

Based on equations (6, 7, and 8), and using Matlab software, the dependence of $\beta$ on $D$, are calculated and plotted. Fig.2 and Fig. 3 show the obtained results.

To realize $10 \mathrm{~m}$ ground resolution distance, $(G R D=\beta H)$ [9], with ETM+ at LandSat-7 orbit $(H=705 \mathrm{~km}), \beta$ should be reduced to $14.18 \mu \mathrm{rad}$. As can be seen from Fig.2, at this value of $\beta, E T M+$ optics diameter will be increased to about $75 \mathrm{~cm}$. While the $H R V$ of SPOT-4 realized the $10 \mathrm{~m}$ resolution, based on normal pushbroom technique, with only $30.91 \mathrm{~cm}$ diameter optics. On the other hand, to realize $5.8 \mathrm{~m}$ ground resolution with 
ETM+ at LandSat-7 orbit, $\beta$ should be reduced to $8.23 \mu$ rad. From Fig.3, at this value of $\beta, E T M+$ optics diameter will be increased to about $169 \mathrm{~cm}$. The PAN camera of IRS-1C realized the $5.8 \mathrm{~m}$ resolution using normal pushbroom technique, with only $22 \mathrm{~cm}$ diameter optics.

From this analysis, we see that to realize high GRD using ETM+ (opto-mechanical scanner), its optics diameter should be increased by about 1.9 times of HRV and about 7.7 times of IRS PAN. This will increase the size and weight of the scanner. On the other side if the optics diameter of HRV and PAN is increased to be as ETM+ $(40.64 \mathrm{~cm})$ a higher GRD can be obtained. This is why high resolution imaging (better than $10 \mathrm{~m}$ ) from space is mandatory based on pushbroom technique.

\subsection{In Case of Normal Pushbroom and TDI Pushbroom Scanners}

From relation (5), for IKONOS TDI, we have: $D=\frac{2.78 \times 10^{-8}}{\beta^{5 / 4}}$

Based on equations (7, 8, and 9), and using Matlab software, the dependence of $\beta$ on $D$, are calculated and plotted. Fig. 4 and Fig. 5 show the obtained results.

To realize $1 \mathrm{~m}$ ground resolution with $H R V$ at SPOT-4 orbit $(H=822 \mathrm{~km}), \beta$ should be reduced to $1.216 \mu \mathrm{rad}$. As can be seen from Fig.4, at this value of $\beta, H R V$ optics diameter will be increased to about $549 \mathrm{~cm}$, which is not practical. On the other hand, to realize $1 \mathrm{~m}$ ground resolution with PAN camera at IRS-1C orbit $(H=816 \mathrm{~km}), \beta$ should be reduced to $1.225 \mu \mathrm{rad}$. As can be seen from Fig.5, at this value of $\beta, P A N$ optics diameter will be increased to about $199 \mathrm{~cm}$, this will increase the size and weight of the scanner. IKONOS TDI realized the $1 \mathrm{~m}$ resolution using the TDI imaging technique, with only $70 \mathrm{~cm}$ diameter optics.

From this result, we see that to realize higher GRD using HRV and PAN (normal pushbroom scanners), its optics diameter should be considerably increased. In HRV, optics diameter should be increased about 7.84 times of IKONS-1 TDI, while in PAN; optics diameter should be increased about 2.84 times of IKONS-1 TDI. This will increase the size and weight of both scanners. This is why very high resolution imaging (better than $2 \mathrm{~m}$ ) from space is mandatory based on TDI pushbroom technique without compromising $\mathrm{S} / \mathrm{N}$.

\subsection{In Case of Opto-mechanical, Normal Pushbroom and TDI Pushbroom Scanners}

Based on equations (6, 7, 8, and 9), and using Matlab software, the dependence of $\beta$ on $D$, are calculated and plotted. Fig.6 and Fig. 7 show the obtained results.

To realize $1 \mathrm{~m}$ ground resolution with ETM+ at LandSat-7 orbit, $\beta$ should $\mathrm{b}$ e reduced to $1.42 \mu \mathrm{rad}$. As can be seen from Fig. 6 and Fig.7, at this value of $\beta, E T M+$ optics diameter 
will be increased to about $2362 \mathrm{~cm}$ (33.74 times of IKONOS-1 TDI) which is not practical. As discussed before, SPOT-4 HRV may realize $1 \mathrm{~m}$ ground resolution from its orbit using normal pushbroom imaging mode with optics diameter $549 \mathrm{~cm}$, and IRS-1C PAN camera may realize $1 \mathrm{~m}$ ground resolution from its orbit, using normal pushbroom imaging mode, with optics diameter $199 \mathrm{~cm}$. IKONOS -1 TDI realized the $1 \mathrm{~m}$ resolution using the TDI imaging technique, with only $70 \mathrm{~cm}$ diameter optics.

Hence using the optics diameter of an opto-mechanical scanner, or even of a normal pushbroom scanner, a higher ground resolution can be produced with a TDI pushbroom scanner, without compromising S/N.

\section{6- CONCLUSION}

Improvement in spatial resolution comes at the expense of $\mathrm{S} / \mathrm{N}$ unless this is offset by a larger aperture, better optical transmission, or increased integration time. Improvements in technology can influence the relative attractiveness of these alternatives. As illustrated above, effective integration time can be increased (and hence $S / N$ ) by adding more detectors and slowing the effective scan rate or by using additional detectors to take multiple samples of the same point in object space and coherently summing the outputs, as in TDI scanner. These strategies offer improved performance at the expense of added complexity in the detector arrays and associated signal processing electronics. On the other hand, technology improvements in these areas have made it possible to exploit such design strategies with relatively low cost and risk.

Also, Improvement in spectral resolution (reducing $\Delta \lambda$ in equation 2) comes at the expense of $\mathrm{S} / \mathrm{N}$ unless this is offset, also, by a larger aperture, better optical transmission, or increased integration time. Therefore another study of the dependence of $\Delta \lambda$ on $\mathrm{D}$ is required. 


\section{REFERENCES}

[1] Slater, P. N., "Remote Sensing - Optics and Optical Systems", AddisonWesley Publishing Company, First edition, 1980.

[2] Kasturirangan, K., "Science and Technology of Imaging From Space", Current Science, Volume 87, Number 5, 10 September 2004.

[3] Joseph, G., "Fundamentals of Remote Sensing", Universities Press, First Edition, 2003.

[4] Larson, W. J., and Wertz, J. R., "Space Mission Analysis and Design", W .J. Larson and Microcosm, Inc., Second Edition, 1992.

[5] Kramer, H .J., "Observation of the Earth and Its Environment - Survey of Missions and Sensors", Springer Verlage Publishing Company, Fourth Edition, 2004.

[6] Joseph, G., "Imaging Sensors for Remote Sensing", Remote Sensing Reviews, Volume 13, pp. 257-342, 1996.

[7] Driggers, R. G., Cox, P., Edwards, T., "Introduction to Infrared and ElectroOptical Systems", Artech House, Inc., First Edition, 1999.

[8] Gaussorgues, G., "IR Thermography", Prentice-Hall International, Inc., First Edition, 1994.

[9] Joseph, G., "How Well Do We Understand Earth Observation Electro-Optical Sensor Parameters?", ISPRS Journal of Photogrammetry and Remote Sensing 55 (2000) 9-12. 

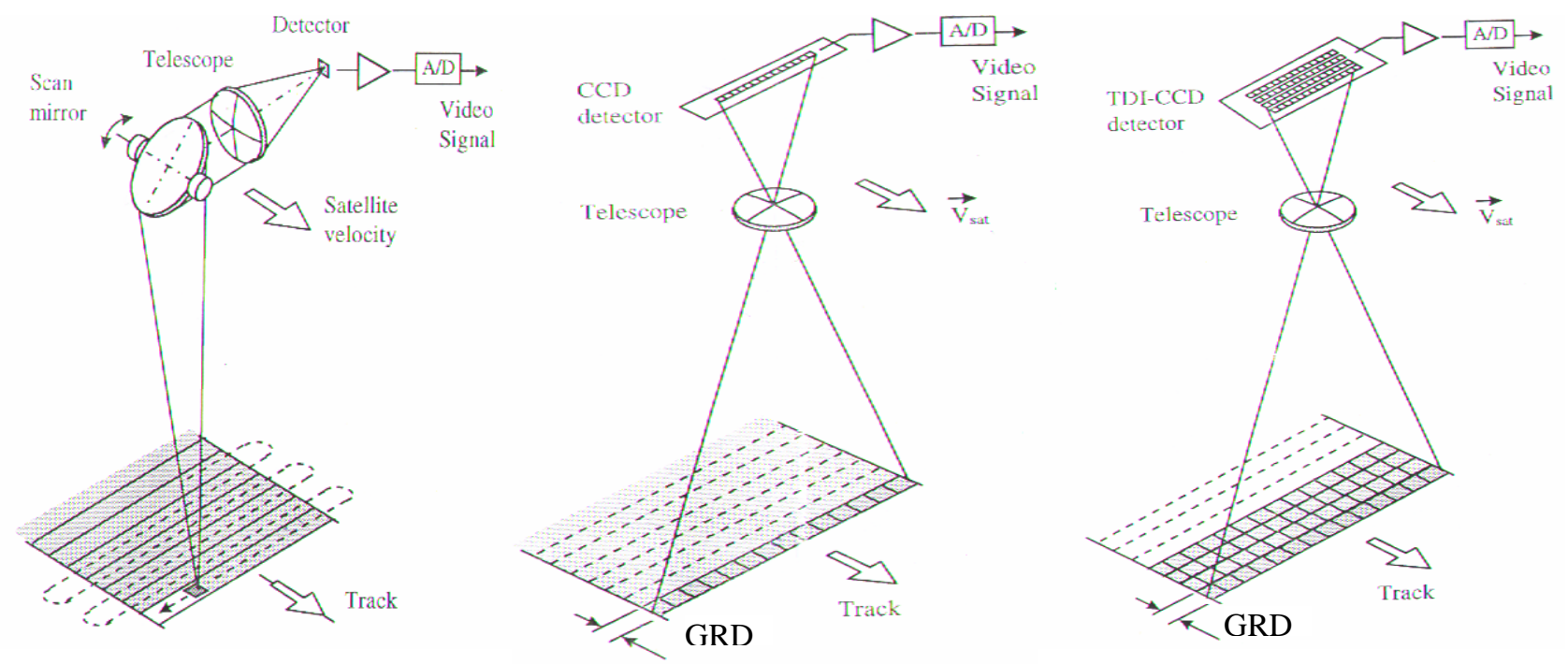

(a) whiskbroom mode

(b) Normal pushbroom mode

(c) Time-delay-and integration

Fig.1. Remote sensors imaging modes

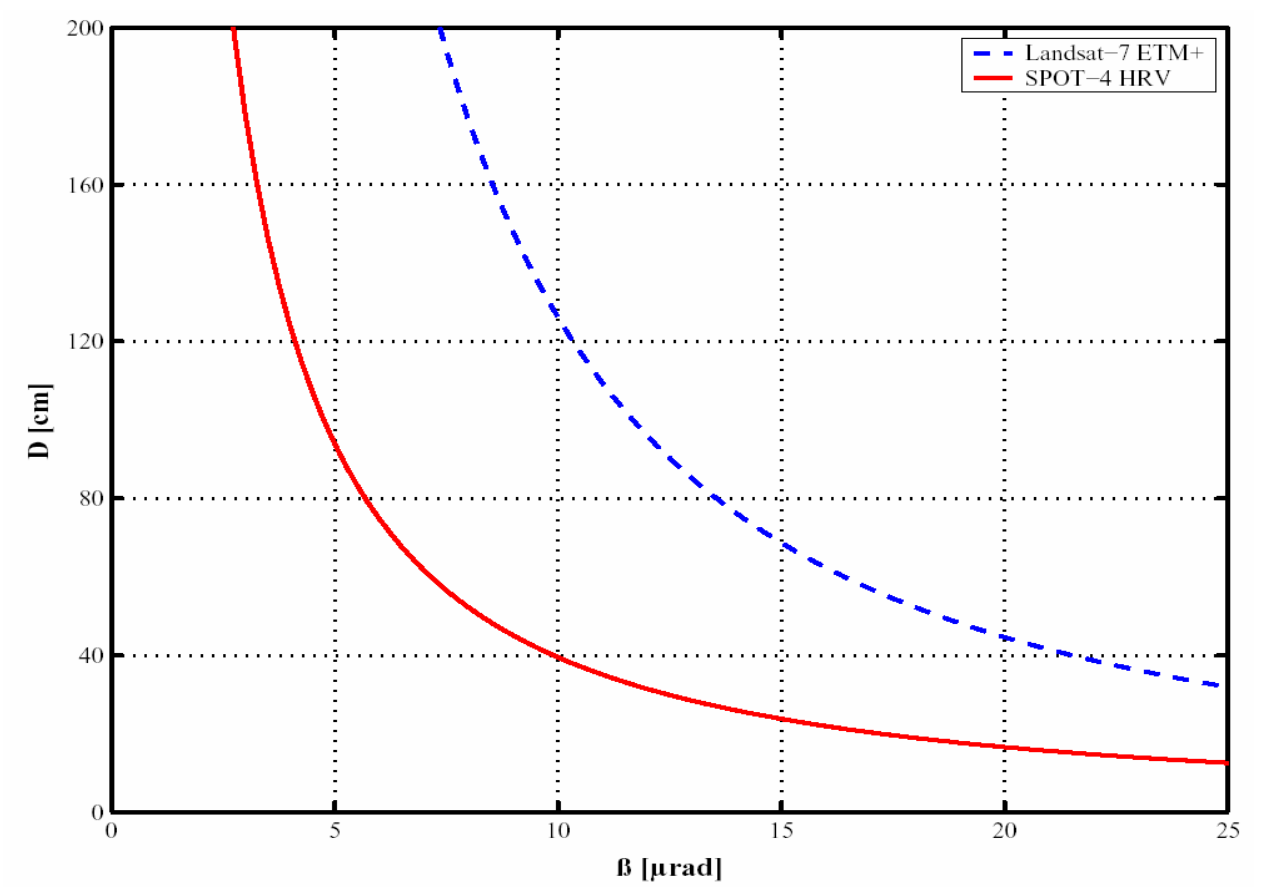

Fig.2. Dependence of $\beta$ on $D$ for LandSat-7 ETM+ and SPOT-4 HRV 


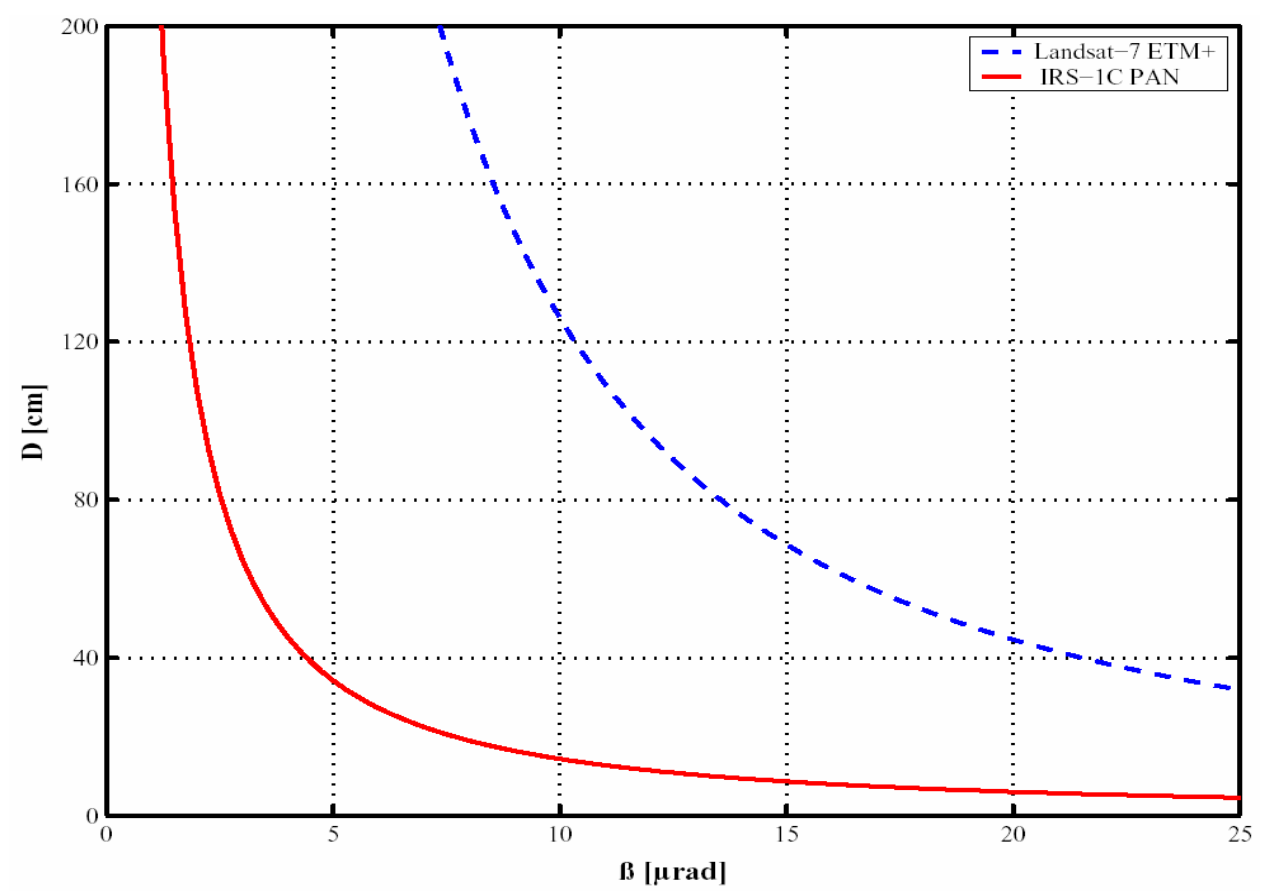

Fig.3. Dependence of $\beta$ on $D$ for LandSat-7 ETM+ and IRS-1C PAN camera

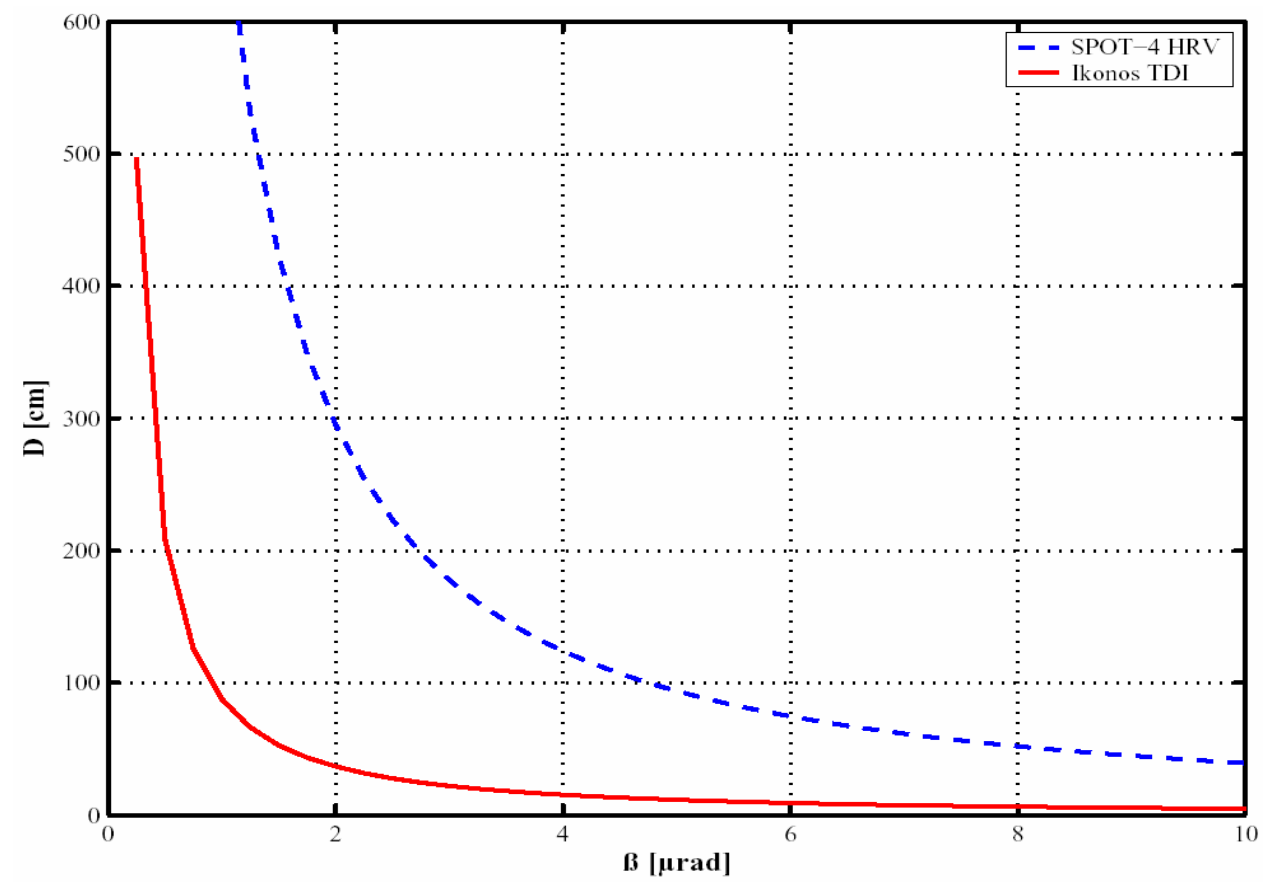

Fig.4. Dependence of $\beta$ on $D$ for SPOT-4 HRV and IKONOS TDI scanners 


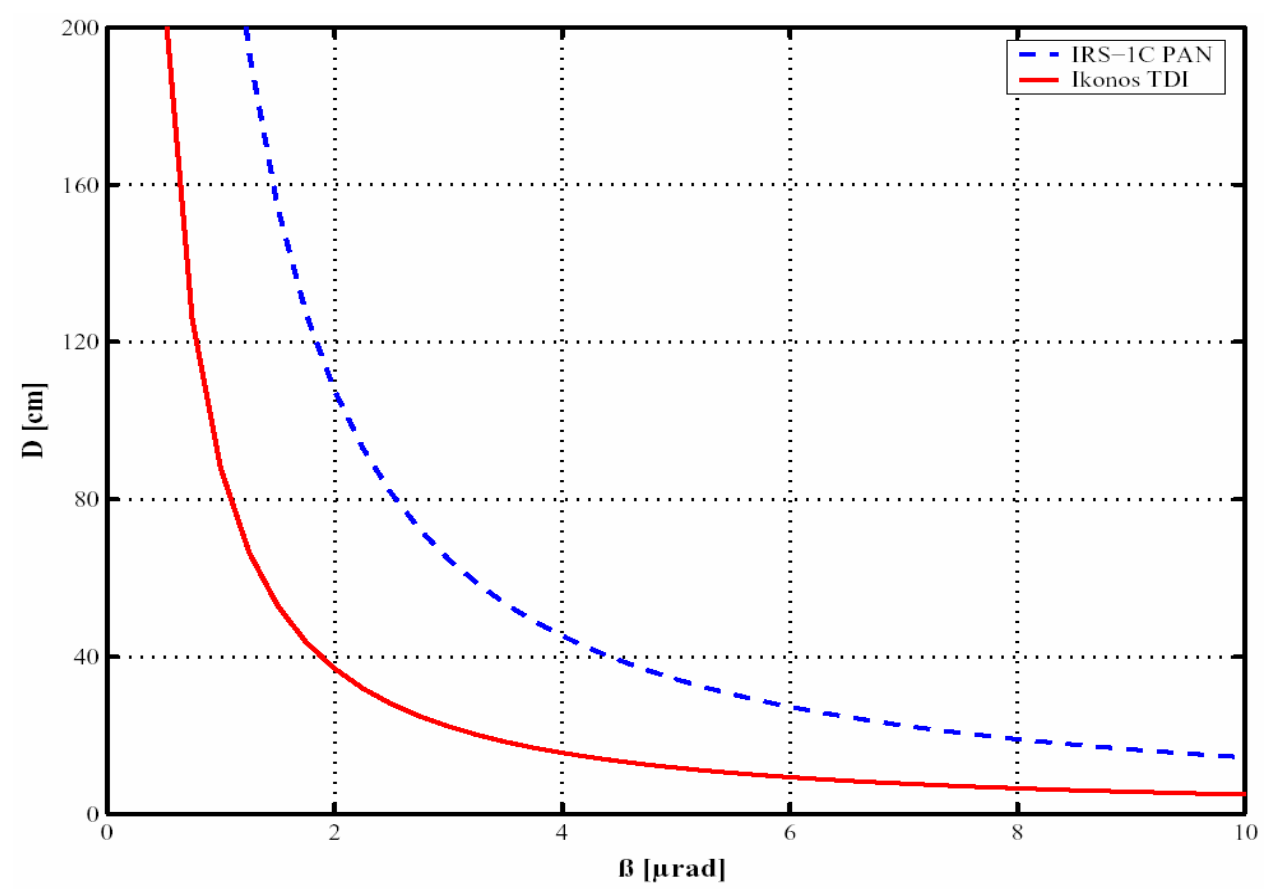

Fig.5. Dependence of $\beta$ on $D$ for IRS -1C PAN and IKONOS TDI scanners

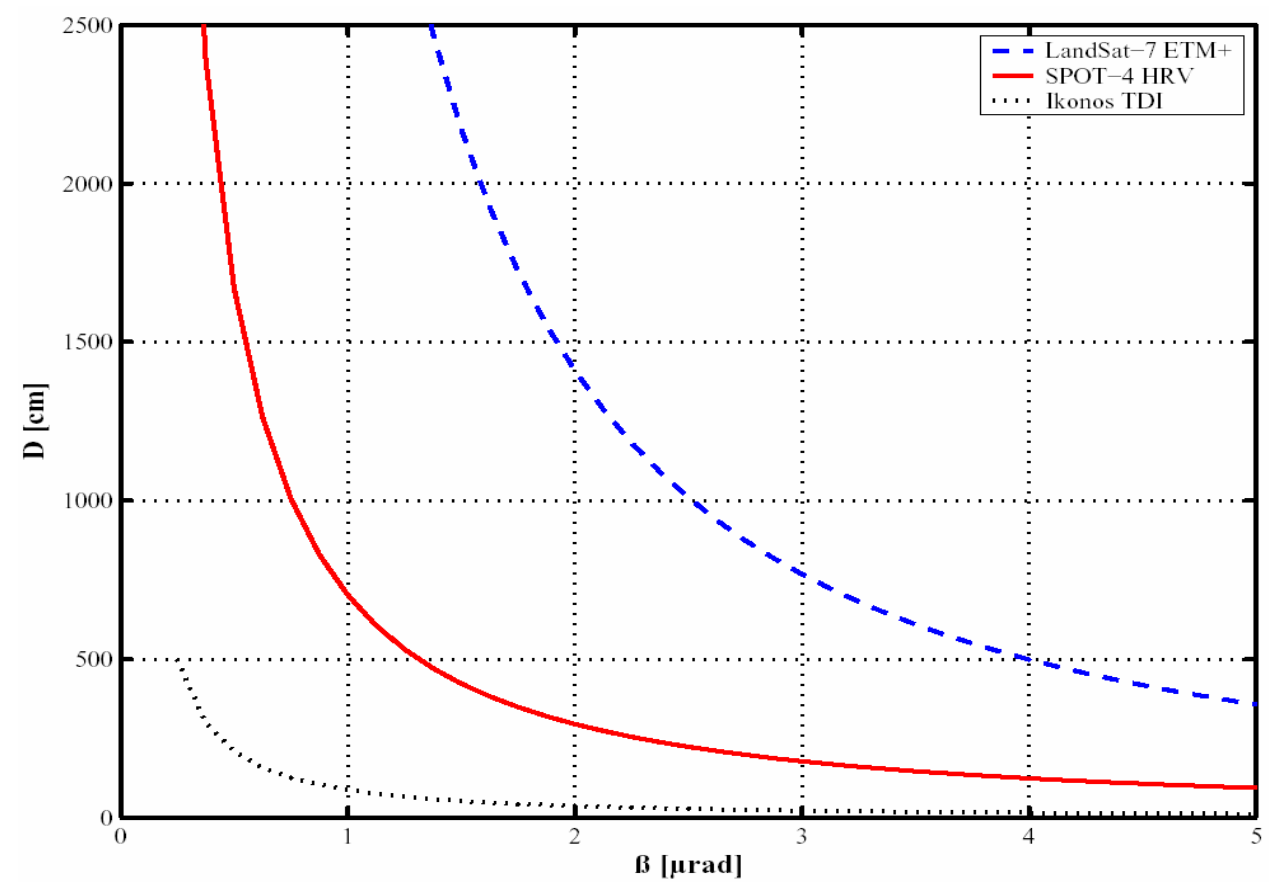

Fig.6. Dependence of $\beta$ on $D$ For LandSat-7 ETM+, SPOT-4 HRV and IKONOS TDI scanners 


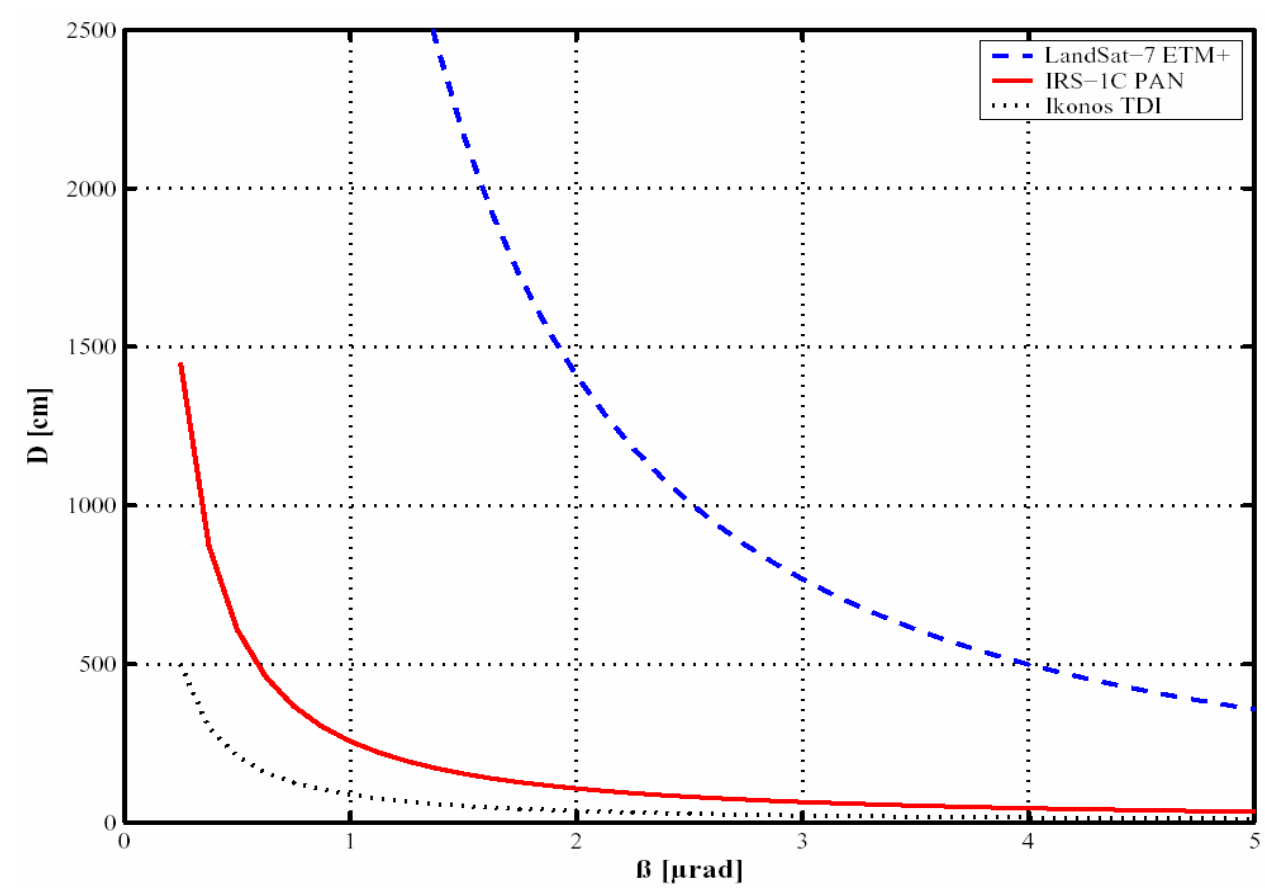

Fig.7. Dependence of $\beta$ on $D$ For LandSat-7 ETM+, IRS-1C PAN and IKONOS TDI scanners 